\title{
Commentary: Axillary artery cannulation for acute type A aortic dissection
}

\author{
Mario Gaudino, MD, and Leonard N. Girardi, MD
}

\author{
From the Department of Cardiothoracic Surgery, Weill Cornell Medicine, New York, NY. \\ Disclosures: Authors have nothing to disclose with regard to commercial support. \\ Received for publication Nov 27, 2018; accepted for publication Nov 28, 2018; available ahead of print Jan 9, \\ 2019. \\ Address for reprints: Mario Gaudino, MD, Department of Cardiothoracic Surgery, Weill Cornell Medicine, 525 \\ East 68th St, New York, NY 10065 (E-mail: mfg9004@med.cornell.edu). \\ J Thorac Cardiovasc Surg 2019;158:660-1 \\ $0022-5223 / \$ 36.00$ \\ Copyright (c) 2018 by The American Association for Thoracic Surgery \\ https://doi.org/10.1016/j.jtcvs.2018.11.125
}

In this issue of the Journal, Dr Svensson and the Cleveland Clinic group ${ }^{1}$ report their 17 -year experience with the repair of acute type A aortic dissection with a particular focus on their switch from femoral to axillary artery cannulation. The series is one of the largest single-center reports published (775 patients, 45/year) and the results are outstanding $(8.8 \%$ operative mortality despite $4.1 \%$ of the patients reaching the operating room in cardiogenic shock, $2.8 \%$ being resuscitated, and $9.3 \%$ presenting with aortic rupture). The authors emphasize the importance of a perfusion strategy individualized to the patient and clinical situation. In $11 \%$ of the cases, the perfusion site was switched or modified intraoperatively because of inadequate perfusion or surgeon's preference.

Switching from peripheral to central cannulation after distal aortic reconstruction is common practice among surgeons even in the absence of signs of malperfusion. Although this seems to be the most common scenario in the Cleveland Clinic experience, in a sizeable percentage of cases, the decision to switch cannulation site is related to detection of high resistance to cardiopulmonary bypass or preferential flow in the false lumen. The excellent outcomes reported by Svensson and colleagues (even in the subset of cases with switched cannulation site) suggest that a high level of vigilance is present in both the surgical and perfusion teams. The ability to rapidly change arterial cannulation site when malperfusion is suspected is a key component to the successful treatment of acute type A aortic dissection.

Because of the per-protocol use of the axillary approach, however, an obvious selection bias exists in favor of the axillary cannulation. As correctly pointed out by the authors, no conclusion can be drawn on the comparison with other perfusion strategies.

Surgical exposure of the axillary artery is not routinely performed by cardiac surgeons. The artery can be deep in the pectoral groove and its wall is fragile and prone to rupture or dissection. The use of a short interposition graft adds additional time but clearly makes cannulation safer.

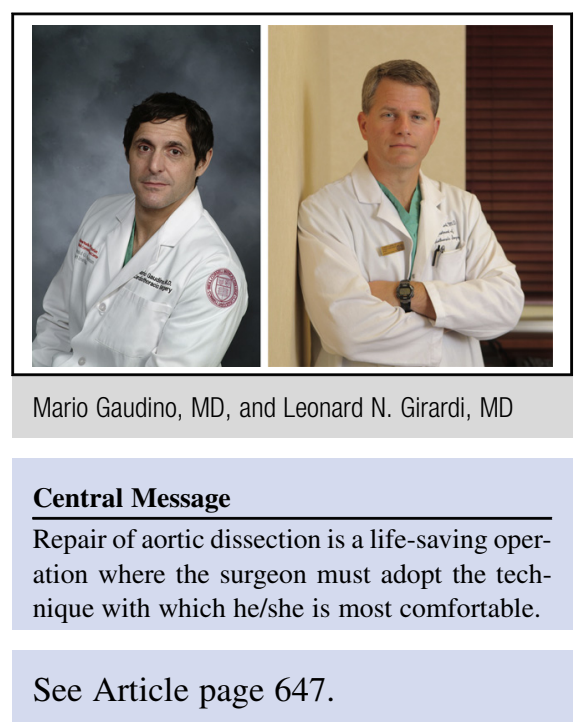

The Cleveland Clinic group does not provide a precise estimate of the time necessary for axillary cannulation, although the authors state in the discussion that it usually adds 15 to 30 minutes to the operation. This is probably much longer at the beginning of the learning curve.

In arch surgery, one important advantage of the axillary artery cannulation is the possibility of switching to antegrade cerebral perfusion during circulatory arrest, a strategy particularly useful when an extended repair and long circulatory arrest time is planned. This was not the case in the present experience as, like us, the Cleveland group rarely adds the risk of complex arch reconstruction to acute type A dissection repair. An overwhelming majority of patients $(77 \%)$ received a limited hemiarch repair with a mean circulatory arrest time of 20 minutes. In a recent European survey on the cannulation strategies used for arch surgery, 54\% the respondents indicated axillary as their cannulation site of preference for acute presentations, ${ }^{2}$ and in a recent report on acute type A repairs from the Society of Thoracic Surgeons database, axillary cannulation was used in almost one third of the cases (27\%; only a few points below femoral cannulation that was used in $36 \%$ of the patients), indicating good acceptance in the surgical community even for emergency cases. ${ }^{3}$

Other sites for antegrade perfusion, such as central aortic or innominate cannulation, have been proposed. ${ }^{4,5}$ For many years, we have adopted routine central direct cannulation of the true lumen, except in very few cases 
when the anatomy of dissection makes central cannulation problematic and in unstable patients.

So, what are the lessons learned from the superb experience presented by Svensson and colleagues? In the hands of highly talented and experienced aortic surgeons, the default use of axillary cannulation for the repair of acute type A aortic dissection is feasible and safe. Most importantly, cannulation site must be tailored to the patient needs and the surgeon's experience and quickly switched if malperfusion is suspected. Repair of acute type A dissections is a life-saving procedure where the operator must adopt the technique with which he/she is most comfortable. Be it femoral, axillary, or central, the goal is to have an alive and awake patient after surgery.

\section{References}

1. Rosinski BF, Idrees JJ, Roselli EE, Germano E, Pasadyn SR, Lowry AM, et al Cannulation strategies in acute type A dissection repair: a systematic axillary artery approach. J Thorac Cardiovasc Surg. 2019;158:647-59.e5.

2. De Paulis R, Czerny M, Weltert L, Bavaria J, Borger MA, Carrel TP, et al EACTS Vascular Domain Group. Current trends in cannulation and neuroprotection during surgery of the aortic arch in Europe. Eur J Cardiothorac Surg. 2015;47:917-23

3. Lee TC, Kon Z, Cheema FH, Grau-Sepulveda MV, Englum B, Kim S, et al Contemporary management and outcomes of acute type A aortic dissection: an analysis of the STS adult cardiac surgery database. J Card Surg. 2018;1: 7-18

4. Reece TB, Tribble CG, Smith RL, Singh RR, Stiles BM, Peeler BB, et al. Central cannulation is safe in acute aortic dissection repair. J Thorac Cardiovasc Surg. 2007;133:428-34.

5. Uchino G, Yunoki K, Sakoda N, Saiki M, Hisamochi K, Yoshida H. Innominate artery cannulation for arterial perfusion during aortic arch surgery. J Card Surg. 2017;32:110-3 